\title{
Aproximación a una valoración cienciométrica de la competitividad
}

\section{Scientometric approach to competitiveness}

\author{
págs. $121-134$ \\ Grupo de Investigación 1: Prospectiva Urbano Territorial \\ Linea de investigación 1: Ciudades competitivas \\ Grupo de Investigación 2: Centro de Investigación en Competitividad Empresarial \\ Línea de investigación 2: Estrategias de Calidad y Competitividad \\ John Fredy Ramírez Ríos•, Angélica María Alzate lbañez••
}

\section{Resumen}

En este artículo se presenta una primera aproximación a una valoración cienciométrica del concepto de competitividad, haciendo uso de los servicios de indexación con funciones bibliométricas de Scopus y Web of Science. Se exponen los criterios de búsqueda, filtrado y clasificación de la producción científica asociada al término y las respectivas mediciones bibliométricas. Los resultados ponen en evidencia los momentos que marcan la evolución histórica, institucional y epistemológica del término, se observa que hasta 1999 el uso del término se relacionaba con temas del desarrollismo económico y del modelo postkeynesiano de producción, bajo el dominio de hegemonías científicas del primer mundo. Hoy en día, la competitividad involucra nuevos ámbitos sociales del desarrollo, bajo el dominio de hegemonías científicas alternativas provenientes de potencias económicas emergentes.

Palabras clave: competitividad, desarrollo, cienciometría, bibliometría,

\begin{abstract}
This paper shows a scientometric approach to competitiveness by using Scopus y Web of Science index services. Scientific production using competitiveness as key term is searched, filtered and classified. The results show key moments of historical, institutional and epistemological evolution of competitiveness through different knowledge areas. Until $2000 \mathrm{com}$ petitiveness was used under post Keynesian model of production and economic developments principles. American and England research universities lead the production. But today, competitiveness is rising in new knowledge fields like social sciences under scientific hegemonies from emerging economies are appearing.
\end{abstract}

Keywords: competitiveness, development, scientometrics, bibliometrics

\footnotetext{
- Sociólogo, Magister en Urbanismo. Docente Investigador. Facultad de Educación Permanente y Avanzada. Fundación Universidad de América. email: john.ramirez@investigadores.uamerica.edu.co

- Ingeniera Química, Magister en Ingeniería, Especialista en Control de Calidad. Docente Investigador. Facultad de Educación Permanente y Avanzada. Fundación Universidad de América. email: angelica.alzate@investigadores.uamerica.edu.co
} 


\section{Introducción}

En el desarrollo de los estados de arte de los proyectos de investigación "Desarrollo de los sistemas de gestión de la calidad. Una visión global: Europa, Asia y América" y "Determinantes del territorio para la competitividad urbana. Una propuesta de conceptualización y medición"; es evidente el uso recurrente del término "competitividad", dentro de los marcos teóricos y conceptuales. Aunque los proyectos pertenecen a disciplinas diferentes como la economía, la administración de empresas, la gestión de la calidad, la gestión ambiental y la planeación territorial, la aparición del término "competitividad" surge como concepto fundamental dentro de esos proyectos en diferentes contextos, vislumbrándose la naturaleza multi, trans e interdisciplinaria de la investigación cientíica. Ello ofrece la oportunidad para el desarrollo de una valoración cienciométrica que permita evidenciar condiciones históricas, institucionales y epistemológicas que este concepto comparte en la producción científica relacionada en variados campos del conocimiento, tales como las ciencias sociales, las ingenierias y las ciencias ambientales.

El cruce de algunos hallazgos preliminares de los resultados del análisis de los estados del arte llevados a cabo para cada uno de los proyectos, abre la posibilidad de un rastreo de los orígenes y evolución del término competitividad, desde un análisis cienciométrico que permita evidenciar su relevancia e interrelación en diferentes ámbitos disciplinares. Con el ánimo de aportar en dicha tarea se propone este artículo corto consistente en una valoración cienciométrica, con base en medición bibliométrica, de la literatura especializada indexada en Scopus $y$ Web of Knowledge que se ha producido desde la década de los sesenta hasta el año 2015, alrededor del término clave "competitiveness".
Requerimientos de la valoración cienciométrica para el análisis de términos científicos: indexación y medición bibliométrica

De acuerdo con Leydesdorff (2001) la cienciometría "can be defined as the study of the quantitative aspects of scientific communication, R\&D practices, and science and technology (S\&T) policies" (p. 13752). En ese sentido, la cienciomeria sirve como herramienta para la resolución de problemas que impliquen la definición de enfoques, lineamientos o marcos de referencia conceptuales en los ámbitos científicos de innovación o de investigación, y desarrollo, no solo en el campo de las ciencias sociales y humanas, sino también de las ciencias de la salud, las ingenierias y las ciencias puras.

La cienciometría se ha convertido en tiempos recientes en un poderoso aliado de instituciones públicas y privadas que fomentan la actividad cientíica, al proveer criterios confiables que pueden ser usados en la construcción de sus planes de política científica o de innovación y en la focalización de sus esfuerzos y recursos de investigación (Nisonger, 2001).

Los primeros ejercicios de evaluación cienciométrica nacieron en el seno del desarrollo de la ciencia norteamericana a finales de la década de los sesenta, en un momento de auge de los sistemas informáticos, de consolidación del trabajo científico colaborativo en grupos de investigación y laboratorios de experimentación, y de producción masiva de artículos y libros de investigación. De ello se deriva el hecho de que toda valoración de ese tipo supone la existencia de un sub-sistema social propio de cientíícos y académicos, creado a partir de la divulgación de sus hallazgos y reflexiones por canales formales de comunicación y redes de colaboración, por ejemplo publicaciones en revistas y libros arbitrados, ponencias y conferencias en eventos, proyectos de extensión, entre otros. Dicho subsistema opera como una comunidad que les permite reconocerse mutuamente, recompensarse por medio de la citación 
LINNEA DE INVESTIGACIÓN: CIUDADES COMPETITIVAS Y ESTRATEGIAS DE CALIDAD Y COMPETITIVIDAD

y la coautoría, jerarquizarse y definir normas de comportamiento; distinguiéndose así de otros sub-sistemas sociales como el político, cultural o religioso.

En las últimas décadas, la cienciometría se ha convertido en un poderoso aliado de instituciones públicas y privadas que fomentan la actividad científica, al proveer criterios confiables que pueden ser usados en la construcción de sus planes de política científica o de innovación y en la focalización de sus esfuerzos y recursos de investigación (Nisonger, 2001). La cienciometría se soporta en servicios de indexación con funciones bibliométricas, cuyo sistema socio-técnico combina de manera inter-dependiente la acción cibernética y la acción humana, mediante soportes especiales de software (Hennemann \& Rybski \& Liefner, 2012; Adams \& Black \& et. al, 2005). Así entonces, la indexación implica un proceso técnico de recolección, clasificación y cuantificación de los flujos de información científica. Al respecto, el primer servicio de indexación de literatura científica que sirvió a la cienciometría se denominó Science Citation Index (SCI), creada por el doctor en lingüística Eugene "Gene" Garfield, y que aún hoy es la más prestigiosa del mundo. EI SCI se convirtió rápidamente en un indicador de las dinámicas del sub-sistema científico norteamericano, midiendo el número de citaciones de los científicos en la literatura especializada existente.

Así se resume la historia de esta primera base de indexación:

In a 1955 article in Science, Dr. Garfield proposed a citation index for science literature. In 1959, the geneticist Dr. Joshua Lederberg began a correspondence with Dr. Garfield following up on the idea of a citation index. Their correspondence led to a meeting with the genetics study section at NIH (National Institutes of Health) and funding to produce a Genetics Citation Index, which would include a multidisciplinary index to the literature of 1961. When NIH and NSF (National
Science Foundation) declined to publish the index, Dr. Garfield's company, now called the Institute for Scientific Information (ISI), began regular publication of the Science Citation Index (SCI) in 1964. SCI distinguished itself from other indexes by being current, comprehensive, and multidisciplinary. Most importantly, the SCI uniquely indexed the references cited in the articles it indexed so users could take advantage of the associations and connections to earlier work that researchers made through the references cited in their papers (Presley \& Caraway, 1999, p. 67).

Hoy en día, se han generado nuevos servicios de indexación con poderosas funciones bibliométricas que han ampliado las posibilidades de los análisis cienciométricos y que, como lo indican Presley \& Caraway (1999), se han convertido en verdaderas herramientas que los académicos puede usar para "identifying relevant papers, avoiding duplications of research, assessing the multidisciplinary influence of their papers, perhaps sparking new ideas through unexpected associations" (p. 68). De esa forma, han permitido "to study the way scientific information is communicated, how the research output of different countries, institutions, and so forth, compares (Presley \& Caraway, 1999, p. 68).

Esta información le permite a la cienciometría, con el necesario soporte estadístico de la bibliometría, dar luces sobre el contexto social que acompaña la creación y evolución de los conceptos propios del conocimiento científico. Los cuales no surgen solamente por el interés de hacer avanzar las fronteras del conocimiento científico, sino que son fruto de un momento histórico, de centros de poder y de instituciones que los agencian, circunstancias que los inscriben en campos disciplinares donde adquieren relevancia. En ese contexto se posicionan hegemonias nacionales, institucionales y discursivas, y los autores como actores del subsistema social científico. De ahí que exista la posibilidad de entender la naturaleza de los conceptos científicos, aproximándose a las formas en que éstos han sido 
producidos y comunicados en el sub-sistema social de la ciencia, por medio de la cienciometría (Merton, 1996).El análisis de esa última información le permite a la cienciometría, con el necesario soporte estadístico de la bibliometría, ofrecer luces sobre el contexto social que acompaña la creación y evolución de los conceptos propios del conocimiento científico; los cuales no surgen solamente por el interés de hacer avanzar las fronteras del conocimiento científico; sino que son fruto, además, de un momento histórico, de centros de poder, de instituciones que los agencian, quienes los inscriben dentro de fronteras disciplinares bajo los cuales adquieren relevancia.

\section{Metodología y alcance de la valoración cienciométrica \\ y de la medición bibliométrica}

Primer paso: definición de objetivos y alcance

La valoración cienciométrica aquí propuesta aborda el contexto que acompaña el surgimiento y la evolución del término "competitividad", a partir de la medición bibliométrica de documentos publicados en fuentes de información indexados como Scopus de Elsevier y Web of Knowledge de Thomson Reuters. El objetivo es evidenciar las condiciones históricas, institucionales y epistemológicas que han moldeado su uso y apropiación en diversos campos disciplinares como la planificación territorial, la gestión de la calidad y la gestión ambiental desde la década de los sesenta.

Las condiciones históricas tienen que ver con el cambio en las dinámicas de producción de las empresas por la expansión intensificada y globalizada de la economía de libre mercado desde la década de los cincuenta hasta el presente, y con el paso de un estado interventor a uno regulador, y la necesidad general de un uso eficiente y eficaz de los recursos en un mundo cada vez más industrializado, urbanizado y poblado. Así mismo las condiciones institucionales están dadas por el posicionamiento de la investigación aplicada y la extensión como funciones misionales de la universidad, inspiradas en el modelo anglosajón de educación superior. Las nuevas sinergias entre empresa, universidad y Estado posibilitan el posicionamiento de centros de investigación privados y públicos, capaces de llevar a cabo investigaciones con alto impacto en el medio social, mediante la generación de conocimiento en repuesta a la solución de problemáticas reales. $Y$, finalmente, las condiciones epistemológicas sustentan la apropiación y significación de nociones científicas en campos disciplinares cada vez más flexibles e interconectados, debido a que el conocimiento científico se basa cada vez menos en el genio individual, y más en una dinámica de intercambio trans-disciplinario entre equipos y grupos de investigación. Esto hace que los conceptos que se derivan de las investigaciones se tornen más complejos y sistémicos, es decir, incorporan paradigmas y formas de ver el mundo que hasta mediados del siglo $X X$ escasamente se relacionaban, y que hoy en día se complementan para una mejor explicación y comprensión de los fenómenos que en él ocurren.

A manera de ejemplo, y como se discutirá más adelante, el caso de la competitividad es una muestra de cómo un término que se usaba estrictamente en el contexto de las ciencias económicas, las ingenierías, los negocios y la administración empresarial ha hecho su camino hasta los ámbitos territoriales y sociales (Martínez, 2008), dando paso al termino de competitividad territorial. Son precisamente ese tipo de evoluciones recientes en el uso de los términos científicos los que fundamentan metodológicamente este tipo de ejercicios analíticos.

Segundo paso: selección del servicio de indexación

En el mercado editorial y de medios existen empresas que cuentan con prestigiosos servicios de indexación, algunos de las cuales incorporan avanzadas funciones bibliométricas. El más reconocido es Web of Knowledge, propiedad de la corporación Thomson Reuters, quien administra hoy en día los índices de citación más prestigiosos del mundo: el original Science Cita- 
tion Index, el Social Science Citation Index, y el Science Citation Index Expanded, entre otros. En Europa, la editorial holandesa Elsevier ha creado Scopus, que según información abierta y oficial de la página, es hoy uno de los servicios de indexación de literatura especializada más grandes del mundo. Contiene aproximadamente 47 millones de registros, algunos de los cuales pueden ser leidos en texto completo a través de la base de datos Science Direct propiedad de la misma editorial ${ }^{1}$.

Scopus y Web of Knwodlege involucran una serie de herramientas inteligentes para rastrear, analizar y visualizar la investigación, permitiendo medir la producción por año, autor(es), filiación institucional, entre otros criterios de búsqueda. Estas bases de datos indexan documentos pro- venientes de cualquier parte del mundo en diferentes idiomas, siempre y cuando se encuentren publicados en fuentes arbitradas y cumplan con los requerimientos definidos por el servicio de indexación. Es importante resaltar que dichos documentos deben contener como requisito el resumen, título y palabras clave en idioma inglés; lo cual permite homologar los contenidos de los artículos entre sí, sin ser una limitante el idioma de la versión original del documento.

Tercer paso: delimitación e identificación de campos y áreas temáticas para la medición bibliométrica

El siguiente paso fue consolidar un universo de documentos sobre los cuales se pudiese ejecutar la medición bibliométrica. La selección

Figura 1.

Criterios de búsqueda general en Scopus

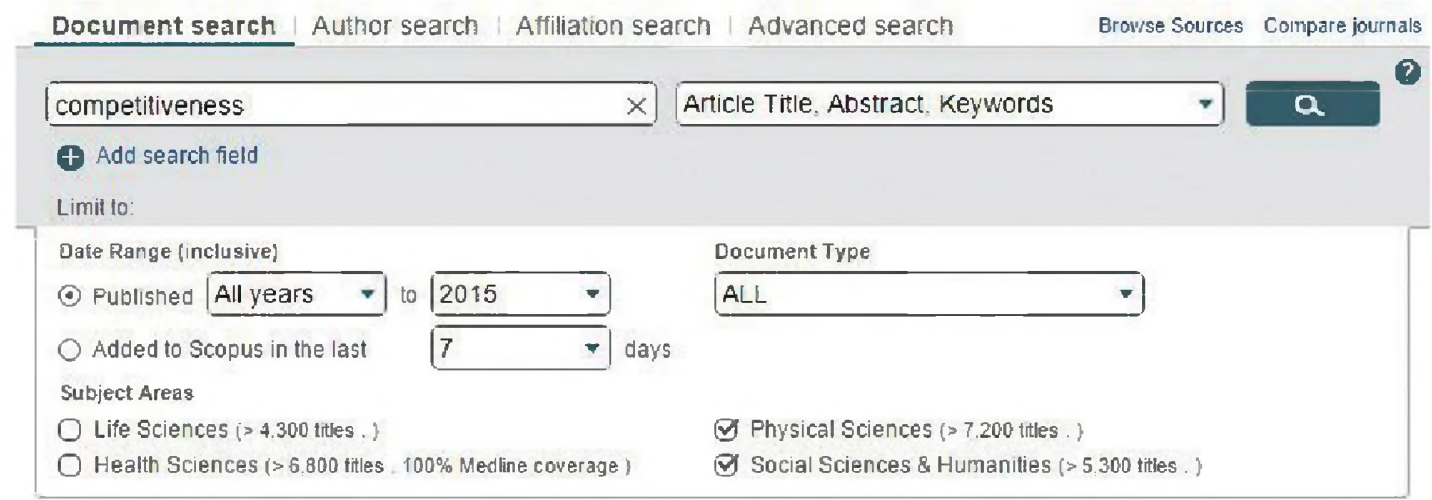

Fuente: Scopus (2016)

1 Existe una fuerte discusión en el ámbito intemacional sobre la "calidad" y la "representatividad" de los documentos referenciados en los servicios de indexación; sectores académicos críticos de los análisis cienciométricos esgrimen válidos argumentos al respecto, afirman por ejemplo, que este tipo de herramientas sirven para legitimar los estándares del que-hacer cientifico propio de las comunidades académicas norte-americanas y europeas, en detrimento de las redes científicas locales que probablemente hagan uso de otro tipo de redes de colaboración, citación y divulgación de sus resultados de investigación. El autor no desconoce dichas limitaciones de estas herramientas de valoración cienciométrica, dejando claro que lo que se pretende es tan solo una primera aproximación. de ese universo se encuentra sujeta a unos criterios de búsqueda que se determinaron previamente de acuerdo al objetivo y alcance del estudio. Así, los criterios de búsqueda iniciales corresponden a los términos y áreas temáticas de interés, durante el periodo de tiempo en el cual se publicaron los documentos y metadatos de filtro, asociados a la información que recopila la base de datos o el servicio de indexación. Dentro de los criterios empleados para realizar la búsqueda general de la producción asociada 
con el término "competitiveness", se incluyeron los siguientes campos del conocimiento "Physical Sciences" y "Social Sciences \& Humanities". En la Figura 1 se encuentran de manera gráfica los criterios de búsqueda iniciales empleados en Scopus.

El sistema arrojó un primer grupo de documentos como resultado de la búsqueda inicial, no obstante, las áreas del conocimiento no son excluyentes en el momento de clasificar un mismo documento, permitiendo a la cienciometria determinar los énfasis discursivos que acom- pañan las conceptualizaciones estudiadas en cada documento. Resultando necesario refinar la búsqueda limitándola a las áreas de mayor relevancia y pertinencia en relación al objetivo del estudio, teniendo en cuentta el carácter fronterizo entre las respectivas áreas, de acuerdo a su contenido e impacto. En la Figura 2. Se presentan los criterios de búsqueda avanzada en concordancia con las áreas del conocimiento, que relacionar el término de competitividad con los procesos de gestión social u organizacional que involucran una intencionalidad humana en el ámbito de la producción de bienes o servicios.

Figura 2.

Areas del conocimiento excluidos según criterios de búsqueda avanzada en Scopus

\begin{tabular}{|c|c|c|c|c|c|c|c|c|}
\hline \multicolumn{9}{|l|}{ Subject Area } \\
\hline Engineering & $(12.572)\}$ & 8 Enemlcal & $\{1.729\}\rangle$ & (8) Immuniclogy and & $(17 n)$ & & & \\
\hline $\begin{array}{l}\text { O Business } \\
\text { Managerrent and } \\
\text { Accountirg }\end{array}$ & $(10.574)\}$ & $\begin{array}{l}\text { Engineering } \\
\checkmark \text { Eartrand Planetary } \\
\text { Setenzes }\end{array}$ & $(1.561)\rangle$ & 8 Heallh Professions & (12) 1) & & & \\
\hline O sacial scences & $(9.3+1)>$ & 8 Ma:hematics & $(1,444)\rangle$ & Nursing & $(54)>$ & & & \\
\hline $\begin{array}{l}D \text { Economics. } \\
\text { Econometrics and }\end{array}$ & $16 \cdot(55)>$ & $\begin{array}{l}8 \text { Arts and } \mathrm{H} \text { umanties } \text { Chemistry }\end{array}$ & $\begin{array}{l}|9021\rangle \\
\mid 901]\rangle\end{array}$ & Of Pliartascience & $\begin{array}{l}(491) \\
(37) \text { ? }\end{array}$ & & & \\
\hline $\begin{array}{l}\text { Fnance } \\
\text { Computer Science }\end{array}$ & $(5,425)>$ & $(9$ Physics and & $\mid 751\}\rangle$ & $\begin{array}{l}\text { Pharmaceutcs } \\
\text { Q Veterinary }\end{array}$ & (9) $)$ & & & \\
\hline $\begin{array}{l}\mathrm{O} \text { Environmental } \\
\text { Science }\end{array}$ & $(4.202)>$ & 8 Psychology & $(735)>$ & Mentstry & (21) & & & \\
\hline (M) Materials Science & $(3,-6,4)\rangle$ & (8) Mutidisciplnary & $(480)>$ & & & & & \\
\hline U Energy & $(2.539)>$ & 8 Mesticine & $|463\rangle\rangle$ & & & & & \\
\hline $\begin{array}{l}\text { Decision Sciences } \\
\text { Agrricultural and } \\
\text { Biologic al Sciences }\end{array}$ & $\begin{array}{l}(2.440)> \\
(1,763)>\end{array}$ & $\begin{array}{l}8 \text { Bio:hemistry. } \\
\text { Genetice snd } \\
\text { Moecular Biblogy }\end{array}$ & $(436)>$ & & & & & \\
\hline Vlew rewer & & & to $5 x$ & ande & & Sut vin. & Fot resvats & - \\
\hline
\end{tabular}

Fuente: Scopus, 2016

El resultado de la búsqueda avanzada arrojó un total de 20.940 documentos, de los cuales el $65.4 \%$ corresponden a artículos, el $18.9 \%$ a documentos de eventos, $5.4 \%$ a capítulos de libro y $4.5 \%$ a revisiones. En la Tabla 1 se presenta una relación de los documentos obtenidos a partir de la búsqueda avanzada en Scopus por área del conocimiento.

A partir de este universo de documentos filtrados y luego de aplicarse todos los criterios de búsqueda, fue posible realizar el análisis bibliométrico empleando las herramientas incorporadas en Scopus. Como criterios de medición bibliométrica y alcance cienciométrico, se tuvieron en cuenta los siguientes aspectos:

a. Año: La producción de literatura especializada se clasifica por fecha de publicación del documento. No hace alusión en modo alguno a la delimitación temporal de la investigación o a la reflexión que se comunica por medio del respectivo documento. Este criterio permite a la cienciometría contar con un criterio estadístico confiable para determinar los momentos de vigencia o surgimiento de determinadas conceptualizaciones en una línea temporal en los respectivos contextos históricos. 
LÍNEA DE INVESTIGACIÓN: CIUDADES COMPETITIVAS Y ESTRATEGIAS DE CALIDAD Y COMPETITIVIDAD

Tabla 1.

Universo de documentos por área del conocimiento

\begin{tabular}{lc}
\hline \multicolumn{1}{c}{ Area del conocimiento } & Documentos \\
\hline Business, Management and & 7.021 \\
Accounting & 6.794 \\
Engineering & 6.765 \\
Social Sciences & 5.037 \\
Economics, Econometrics and & 1.688 \\
Finance & 20.940 \\
\hline Environmental Science & \\
\hline Total &
\end{tabular}

b. Filiación institucional: Se clasifica la producción de literatura especializada por la filiación institucional del autor al momento de publicar el documento, en los casos en que el documento es en coautoría, el sistema reporta por cada autor la respectiva filiación. Este aspecto le permite a la cienciometría indagar el liderazgo de las instituciones universitarias, gubernamentales $y$ de investigación en el estudio del término valorado.

c. Países: La filiación institucional se encuentra asociada a los paises, permitiendo hacer un análisis sobre los países predominantes o emergentes en las diferentes disciplinas y áreas del conocimiento. No obstante, el peso que tiene la producción científica generada en países con inversiones per cápita en ciencia y tecnología altas, y que estimulan a sus científicos para que publiquen en revistas prestigiosas, incide en los resultados arrojados por la base de datos, generando una percepción subjetiva al realizar el análisis de la documentación producida en una reducida cantidad de países.

\section{Resultados de la medición bibliométrica y discusión de valoración cienciométrica}

En esta sección se presentan los resultados de búsqueda dentro de la colección de Scopus, haciendo uso de las herramientas bibliometricas dispuestas por este servicio de indexación, seguido de una discusión cienciométrica. Desde el año 1966 ha sido reportada la producción aso- ciada con el término de competitividad; inicialmente el comportamiento de la producción fue baja e irregular hasta el año 1989, en donde se evidencia un crecimiento marcado en la produccióin, incrementándose de manera exponencial a partir del año 2000 (ver Figura 3). Por otra parte, la Figura 4 ejemplifica la naturaleza de las hegemonías nacionales asociadas a esa producción alrededor del término, evidenciándose el liderazgo indiscutible de los países del primer mundo en aquel momento.

Esta primera medición bibliométrica revela el interés creciente que ha despertado el concepto de competitividad desde comienzos de la década de los sesenta, surgiendo las primeras raíces en los ámbitos temáticos propios del comercio internacional y del desarrollo industrial. Los primeros cuatro documentos indexados en Scopus que hicieron uso de manera explícita el concepto de competitividad, se enfocaron en los siguientees temas, a saber: Tiresome subsidies - the nuisance of subsidies distorting international competition, The maritime telematics strategy in the European communities, Computer control chemical plant - design and assessment framework y Antwerp invests to beat competition.

En la tabla 2 se observa que en los primeros momentos de uso del término competitividad en la producción científica, éste aparece implícitamente relacionado con el concepto de desarrollo centrado en el crecimiento económico, y la necesidad de afrontar directamente los nuevos problemas de competencia entre las industrias nacionales. Al respecto es necesario indicar, que el orden geopolítico y económico capitalista mundial en el tercer cuarto del siglo XX se basó en una lógica denominado post-keynesian competition states (Brenner, 2004, p. 172), según la cual las industrias nacionales debian empezar a reconsiderar las condiciones de administración de las industrias nacionales debían empezar a reconsiderar las condiciones de su administración, de operación de la producción, y de marketing de sus productos. 
Figura 3.

Producción anual de documentos indexados en Scopus durante el periodo 1966-1999: Competitividad

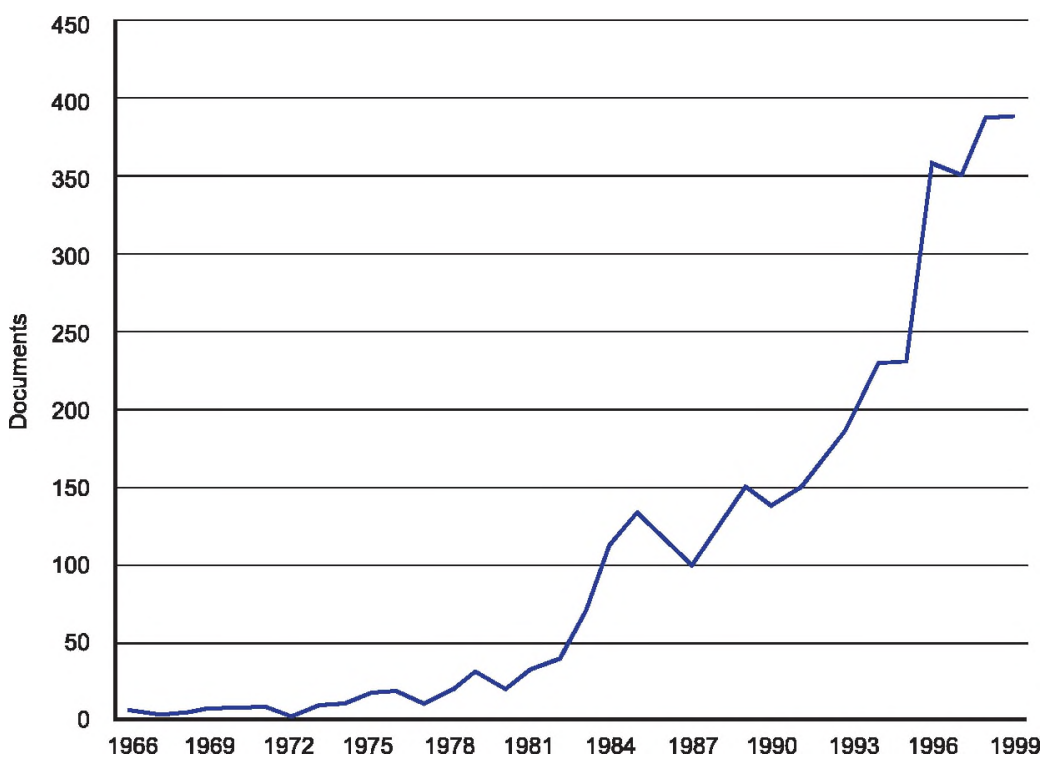

Fuente: Scopus, 2016

Figura 4.

Producción por países de documentos indexados en Scopus durante el periodo 1966-1999 en relación al término "Competitividad"

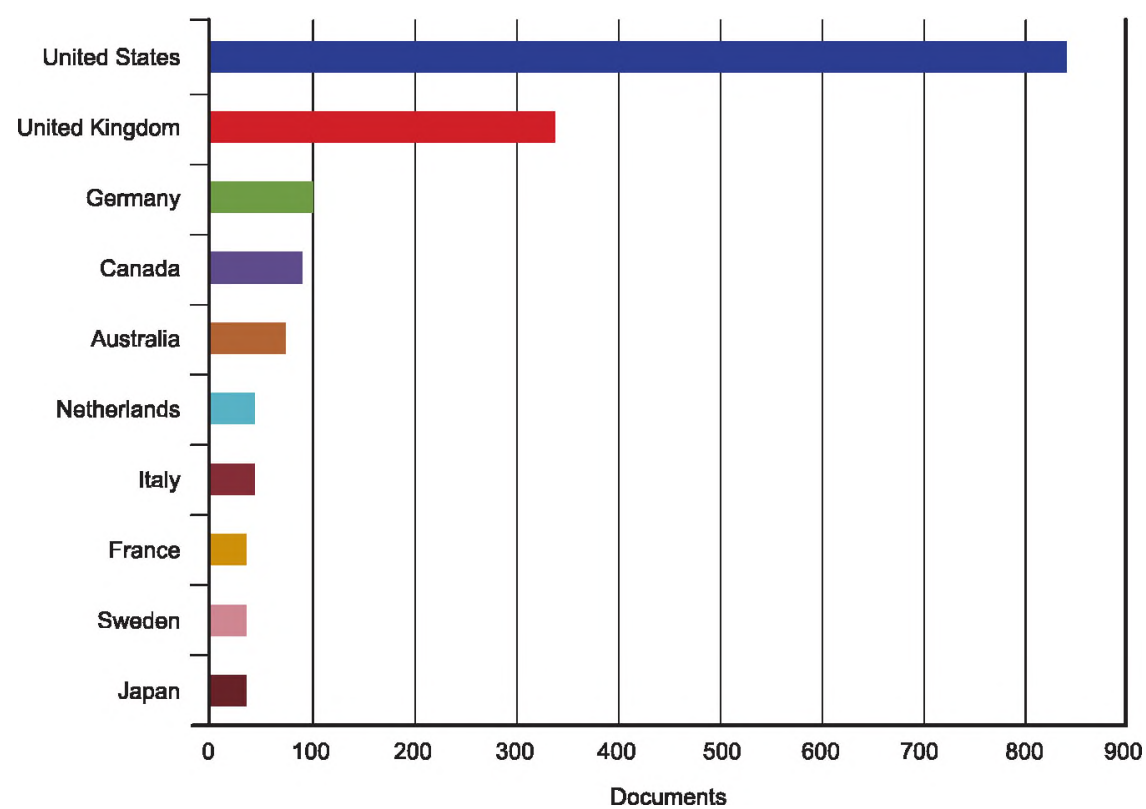

Fuente: Scopus, 2016 
LÍNEA DE INVESTIGACIÓN: CIUDADES COMPETITIVAS Y ESTRATEGIAS DE CALIDAD Y COMPETITIVIDAD

Tabla 2.

Producción de documentos indexados en Scopus por recurso durante el periodo 1966-1999 en

Competitividad

\begin{tabular}{|c|c|c|}
\hline Recurso & Area del conocimiento & Documentos \\
\hline $\begin{array}{l}\text { SAE Technical } \\
\text { Papers }\end{array}$ & $\begin{array}{l}\text { Engineering: Automotive Engineering, Industrial and Manufacturing } \\
\text { Engineering, Safety, Risk, Reliability and Quality } \\
\text { Environmental Science: Pollution }\end{array}$ & 56 \\
\hline $\begin{array}{l}\text { World Develop- } \\
\text { ment }\end{array}$ & $\begin{array}{l}\text { Social Sciences: Development, Geography, Planning and Development, } \\
\text { Sociology and Political Science } \\
\text { Business, Management and Accounting: Management of Technology }\end{array}$ & 41 \\
\hline Technovation & $\begin{array}{l}\text { and Innovation } \\
\text { Engineering } \\
\text { Business, Management and Accounting: Management of Technology }\end{array}$ & 36 \\
\hline Research Policy & $\begin{array}{l}\text { and Innovation, Strategy and Management } \\
\text { Decision Sciences: Management Science and Operations Research } \\
\text { Engineering }\end{array}$ & 33 \\
\hline $\begin{array}{l}\text { Textile Outlook } \\
\text { International }\end{array}$ & $\begin{array}{l}\text { Business, Management and Accounting: Business, Management and } \\
\text { Accounting (miscellaneous) } \\
\text { Business, Management and Accounting: Strategy and Management }\end{array}$ & 32 \\
\hline $\begin{array}{l}\text { Long Range } \\
\text { Planning }\end{array}$ & $\begin{array}{l}\text { Economics, Econometrics and Finance: Finance } \\
\text { Social Sciences: Geography, Planning and Development }\end{array}$ & 31 \\
\hline $\begin{array}{l}\text { IEE Colloquium } \\
\text { Digest }\end{array}$ & Engineering: Electrical and Electronic Engineering & 27 \\
\hline Regional Studies & $\begin{array}{l}\text { Environmental Science } \\
\text { Social Sciences }\end{array}$ & 25 \\
\hline $\begin{array}{l}\text { Jtn Weekly } \\
\text { International }\end{array}$ & $\begin{array}{l}\text { Business, Management and Accounting: Business and International } \\
\text { Management } \\
\text { Business, Management and Accounting }\end{array}$ & 24 \\
\hline $\begin{array}{l}\text { Journal of Pro- } \\
\text { duction Econo- } \\
\text { mics }\end{array}$ & $\begin{array}{l}\text { Decision Sciences: Management Science and Operations Research } \\
\text { Economics, Econometrics and Finance: Economics and Econometrics } \\
\text { Engineering: Industrial and Manufacturing Engineering } \\
\text { Business, Management and Accounting: Business and International }\end{array}$ & 24 \\
\hline $\begin{array}{l}\text { Technology in } \\
\text { Society }\end{array}$ & $\begin{array}{l}\text { Management } \\
\text { Social Sciences: Education, Human Factors and Ergonomics, Sociology } \\
\text { and Political Science } \\
\text { Business, Management and Accounting: Business, Management and }\end{array}$ & 24 \\
\hline Intereconomics & $\begin{array}{l}\text { Accounting (miscellaneous) } \\
\text { Economics, Econometrics and Finance: Economics, Econometrics and } \\
\text { Finance (miscellaneous) }\end{array}$ & 23 \\
\hline $\begin{array}{l}\text { Ekonomicky } \\
\text { Casopis }\end{array}$ & Economics, Econometrics and Finance: Economics and Econometrics & 23 \\
\hline
\end{tabular}

Fuente: Scopus, 2016 
Hasta ese momento y desde una perspectiva cienciométrica, la competitividad se exhibe como un asunto relacionado directamente con la búsqueda de aumentar la productividad y las ventajas comparativas (Krugman, 1994;Kitson et al., 2004), por lo que se puede afirmar que el término estaba vinculado directamente con una visión economicista del desarrollo. En la teoría económica clásica la productividad hacía referencia al grado de eficiencia de la producción de una empresa, en cuanto a su capacidad de producir más en menos tiempo. Según Porter (2008, citado en IMD World Competitiveness Center, 2014, p. 492) es posible considerar que la "competitiveness would become the productivity with which a nation utilizes its human, capital and natural resources", tratándose no solo la productividad competitiva que relaciona las variables llanas de trabajo y tiempo, sino de una serie de variables adicionales como la tecnología, educación y sistema de valores.

Esa lógica economicista y empresarial fundamentó todo discurso proclive a la competitividad hasta el siglo XXI. De hecho, el World Competitiveness Center, sostiene que la "competitividad" es un "campo de la teoría económica" y corresponde a una expresión empleada para la creación de valor económico en las empresas (IMD World Competitiveness Center, 2014, p. 492).

La Tabla 3 muestra que a comienzos del siglo, el término competitividad sufre un cambio de énfasis relacionado con nuevas problemáticas del desarrollo, y no solo economicista, sino a nivel de producto, de calidad, de producción regional y de turismo. En la Figura 5 se observa el surgimiento de nuevas hegemonías nacionales: China y Taiwan, aunque sin perder el protagonismo de la hegemonía americana. A pesar de que se evidencia el crecimiento de publicaciones provenientes de oriente, las universidades de Europa y EE-UU continúan dominando la producción alrededor del término de competitividad, liderando el interés conceptual por la competitividad urbana, dada su solidez y tradición institucional que opacan, por ejemplo, los incipientes esfuerzos de las universidades chinas.

Figura 5.

Producción anual de documentos indexados en Scopus durante el periodo 2000-2015: "Competitividad"

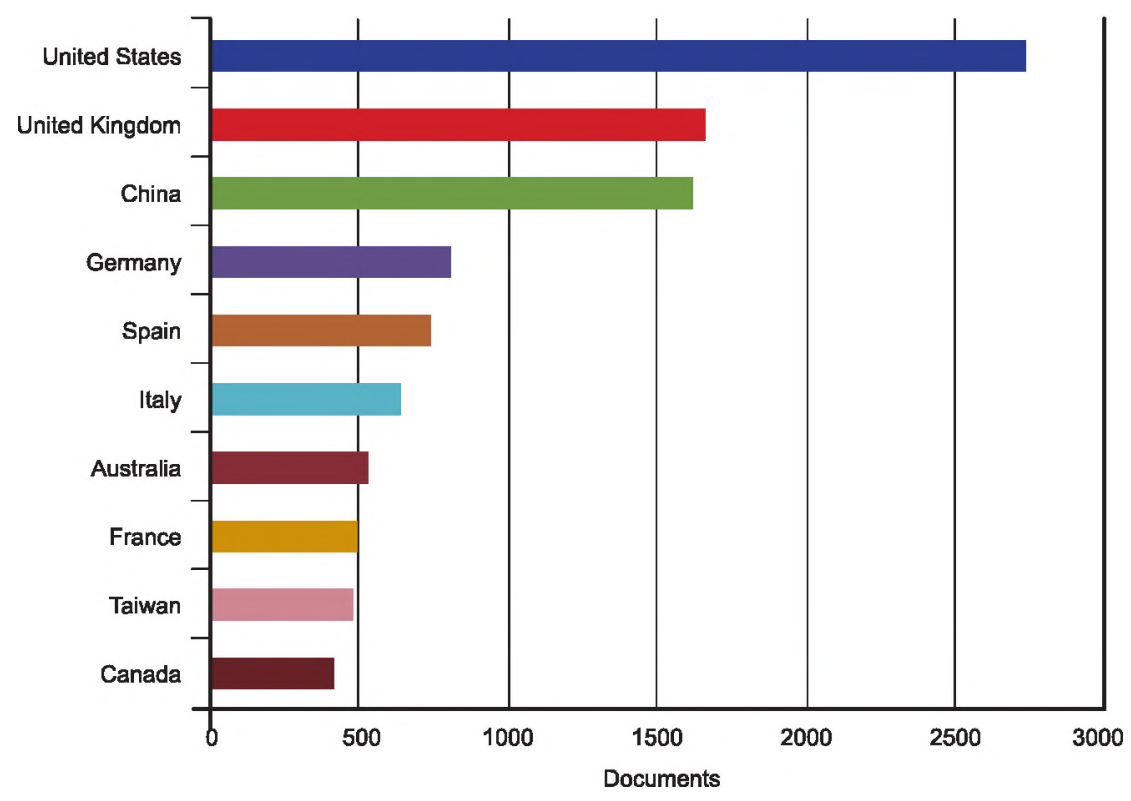

Fuente: Scopus, 2016 
LÍNEA DE INVESTIGACIÓN: CIUDADES COMPETITIVAS Y ESTRATEGIAS DE CALIDAD Y COMPETITIVIDAD

Tabla 3.

Producción de documentos indexados en Scopus por recurso durante el periodo 2000-2015 en Competitividad

\begin{tabular}{|c|c|c|}
\hline Recurso & Área del Conocimiento & Documentos \\
\hline Advanced Materials Research & Engineering & 310 \\
\hline $\begin{array}{l}\text { Applied Mechanics and Mate- } \\
\text { rials }\end{array}$ & Engineering & 272 \\
\hline Actual Problems of Economics & $\begin{array}{l}\text { Economics, Econometrics and Finance: Economics and } \\
\text { Econometrics } \\
\text { Business, Management and Accounting: Business and }\end{array}$ & 268 \\
\hline Quality Access to Success & $\begin{array}{l}\text { International Management, Management Information Sys- } \\
\text { tems, Strategy and Management }\end{array}$ & 110 \\
\hline European Planning Studies & Social Sciences: Geography, Planning and Development & 108 \\
\hline Textiles South East Asia & $\begin{array}{l}\text { Business, Management and Accounting: Business, Mana- } \\
\text { gement and Accounting (miscellaneous) }\end{array}$ & 96 \\
\hline Regional Studies & $\begin{array}{l}\text { Environmental Science } \\
\text { Social Sciences }\end{array}$ & 88 \\
\hline Economic Annals XXI & $\begin{array}{l}\text { Economics, Econometrics and Finance } \\
\text { Social Sciences: Sociology and Political Science }\end{array}$ & 86 \\
\hline Applied Economics & $\begin{array}{l}\text { Economics, Econometrics and Finance: Economics and } \\
\text { Econometrics }\end{array}$ & 84 \\
\hline Tourism Management & $\begin{array}{l}\text { Business, Management and Accounting: Strategy and Ma- } \\
\text { nagement, Tourism, Leisure and Hospitality Management } \\
\text { Social Sciences: Development, Transportation }\end{array}$ & 79 \\
\hline $\begin{array}{l}\text { Lecture Notes in Electrical } \\
\text { Engineering }\end{array}$ & Engineering: Industrial and Manufacturing Engineering & 78 \\
\hline SAE Technical Papers & $\begin{array}{l}\text { Engineering: Automotive Engineering, Industrial and Manu- } \\
\text { facturing, Safety, Risk, Reliability and Quality } \\
\text { Environmental Science: Pollution }\end{array}$ & 75 \\
\hline Ekonomicky Casopis & $\begin{array}{l}\text { Economics, Econometrics and Finance: Economics and } \\
\text { Econometrics }\end{array}$ & 73 \\
\hline Service Industries Journal & $\begin{array}{l}\text { Business, Management and Accounting: Management of } \\
\text { Technology and Innovation, Strategy and Management } \\
\text { Business, Management and Accounting: Management of }\end{array}$ & 71 \\
\hline Technovation & $\begin{array}{l}\text { Technology and Innovation } \\
\text { Engineering }\end{array}$ & 68 \\
\hline Textile Outlook International & $\begin{array}{l}\text { Business, Management and Accounting: Business, Mana- } \\
\text { gement and Accounting (miscellaneous) } \\
\text { Business, Management and Accounting: Business and } \\
\text { International Management }\end{array}$ & 58 \\
\hline Engineering Economics & $\begin{array}{l}\text { Economics, Econometrics and Finance: Economics and } \\
\text { Econometrics } \\
\text { Engineering: Engineering (miscellaneous) } \\
\text { Business, Management and Accounting: Management }\end{array}$ & 56 \\
\hline $\begin{array}{l}\text { Construction Management and } \\
\text { Economics }\end{array}$ & $\begin{array}{l}\text { Information Systems } \\
\text { Engineering: Building and Construction, Industrial and } \\
\text { Manufacturing Engineering }\end{array}$ & 54 \\
\hline
\end{tabular}

Fuente: Scopus, 2016 
La existencia de otros servicios e indexación como Web of Science, permite ampliar el espectro de información para realizar análisis cienciométrico, a partir de la medición bibliométrica de las publicaciones en periodos y áreas determinadas de acuerdo al objeto de estudio y en particular para el desarrollo de estados del arte. En la Figura 6 se presenta una relación del número de documentos por área del conocimiento arrojados a partir de la búsqueda en
Web of Science siguiendo los criterios de este estudio en particular. Como se observa en la gráfica, es posible seleccionar categorlas especificas en diferentes áreas del conocimiento, lo cual da como resultado de búsqueda inicial un total de 5.301 documentos. Las áreas de economia, negocios y administración ocupan las posiciones con mayor porcentaje de representatividad en relación al uso del término competitividad.

Figura 6.

Relación de documentos por áreas del conocimiento según criterios de búsqueda avanzada en Web of Science

\begin{tabular}{|c|c|c|c|c|}
\hline $\begin{array}{l}\rightarrow \text { View Records } \\
\text { × Exelude Records }\end{array}$ & Field: Web of Science Categories & Record Count & $\%$ of 5301 & Bar Chart \\
\hline$\square$ & ECONOMICS & 2125 & $40.087 \%$ & 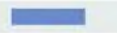 \\
\hline 已 & MANAGEMENT & 1478 & $27.882 \%$ & $=$ \\
\hline 0 & BUSINESS & 910 & $17.167 \%$ & a \\
\hline ○ & OPERATIONS RESEARCH MANAGEMENT SCIENCE & 725 & $13.677 \%$ & - \\
\hline$\square$ & ENVIRONMENTAL STUDIES & 707 & $13.337 \%$ & - \\
\hline E & ENGINEERING INDUSTR|AL & 575 & $10.847 \%$ & 口 \\
\hline ○ & ENGINEERING MANUFACTURING & 439 & $8.281 \%$ & - \\
\hline D & PLANNING DEVELOPMENT & 390 & $7.357 \%$ & घ \\
\hline D & ENVIRONMENTAL SCIENCES & 278 & $5.244 \%$ & - \\
\hline D & URBAN STUDIES & 206 & $3.886 \%$ & I \\
\hline
\end{tabular}

Fuente: Scopus, 2016

\section{Comentarios}

La valoración cienciométrica de "competitividad", permite entrever los periodos históricos que han marcado el surgimiento y evolución del concepto en diferentes contextos, así como las instituciones y los desarrollos conceptuales, que permiten demostrar la vinculación entre la primera aparición del concepto de competitividad en la década de los sesenta, con las nuevas lógicas de desarrollo economicista del capitalismo global, el papel de las industrias y del estado nacional. El posicionamiento palpable cada vez más fuerte de China y demás paises emergentes en la producción cientifica relacionada con temas competitividad, sin relegar el papel hegemónico de instituciones de investigación en Europa y Estados Unidos.
Se observa un periodo de transición en el cual la competitividad entendida como un asunto meramente económico, evoluciona a una comprensión contemporánea mucho más holística en términos sociales y políticos, con un liderazgo cada vez más creciente de las ciencias sociales. A principios de los noventa con la demanda de desarrollo humano y social en contextos territoriales más específicos y localizados en el territorio, dando paso a un concepto de competitividad que involucra una preocupación por las dimensiones sociales y humanas del desarrollo, llegando finalmente a un término más complejo denominado "competitividad territorial".

Dentro de los principales autores que han aportado al surgimiento y evolución del con- 
LIINEA DE INVESTIGACIÓN: CIUDADES COMPETITIVAS Y ESTRATEGIAS DE CALIDAD Y COMPETITIVIDAD

cepto de competitividad en las áreas del conocimiento relacionadas con el objetivo del presente estudio, se destacan Porter, M.E. (1980,1985), Buckley P.J., (1988), Cohen, W.M. (1990) y Krugman,P. $(1994,1996)$ en el ámbito de la gestión. Cohen, W.M. (1990) resalta la importancia del papel de cada una de las especialidades dentro de una organización, la capacidad de absorción y se pone en manifiesto la necesidad de la innovación para la competitividad. Por su parte, Barney, J. (1991) aporta al área la gestión estratégica de las organizaciones, examinando la potencialidad de los recursos para la generación de ventajas competitivas sostenibles. Finalmente, Krugman,P. $(1994,1996)$ presenta una serie de reflexiones y debates alrededor de la idea de la competitividad en países como Estados Unidos y su relación con algunos factores específicos.

En el ámbito de la planeación territorial urbana, dentro de los principales exponentes se resaltan Begg, I. (1999), Crouch, G.I. (1999), Storper, M. (1997), Kitson, M. (2004). Begg, I. (1999) presenta un documento en donde se plantea el cómo desde diferentes niveles las ciudades empiezan a competir en un mundo globalizado, partiendo de que la estrategia de desarrollo económico inicia con la premisa de "something can be done".

A partir de las reflexiones basadas en valoración cienciométrica sobre el término competitividad, se ha evidenciado la utilidad de la cienciometría y de las mediciones bibliométricas, para el análisis y la comprensión general de ciertas dinámicas cientificas que se generan alrededor de términos especializados, lo que podría llegar a aportar en la creación de estados del arte robustos y coherentes con el desarrollo del que-hacer científico a nivel internacional.

El campo vasto de indagación que todavía existe alrededor del término de competitividad, le otorga una renovada legitimidad académica a los proyectos de la Fundación Universidad de América, y que se están desarrollando en los diversos campos de conocimiento de competencia de la universidad. Además, se abren posibilidades de cooperación inter y trans-disciplinar entre los grupos de investigación que lideran dichos proyectos.

\section{Referencias}

Adams, S. J. D., Black, G. C, Clemmons, J. R., Paula, E., \& Stephan, P. E. (2005). Scientific teams and institutional collaborations: Evidence from U.S. Universities, 1981-1999. Research Policy, 34(3), 259-285.

Barney, J. (1991). Firm resources and sustained competitive advantage.Journal of management, 17(1), 99-120.

Begg, I. (1999). Cities and competitiveness. Urban studies, 36(5/6), 795.

Breheny, M.J. (Ed) (1992) Sustainable development and urban form. London, Pion Limited

Brenner, N. (2004). Interlocality competition as a state project: Urban locational policy and the rescaling of state space. En: New State Spaces: Urban Governance and the Rescaling of Statehood. Oxford: Oxford University Press. p. 172-256.

Buckley, P. J., Pass, C. L., \& Prescott, K. (1988). Measures of international competitiveness: a critical survey*†. Journal of marketing management, 4(2), 175-200. 
Castells, M. (1996) Rise of the Network Society (Information Age Series). New York: Wiley-Blackwell

Cohen, W. M., \& Levinthal, D. A. (1990). Absorptive Capacity: A New Perspective on Learning and Innovation. Administrative Science Quarterly,35(1), 128-152.

Guimardes, R.P. (1994) El Desarrollo Sustentable: ¿Propuesta Alternativa o Retórica Neoliberal? Eure. 20(61), 41-56.

Hennemann, S; Rybski, D; Liefner, I. (2012) The myth of global science collaboration-Collaboration patterns in epistemic communities Journal of Informetrics, 6(2), 217-225.

IMD WORLD COMPETITIVENESS CENTER. Imd world competitiveness yearbook 2014. Switzerland.

Kitson, M., Martin, R., \& Tyler, P. (2004). Regional competitiveness: an elusive yet key concept?. Regional studies, 38(9), 991-999.

Krugman, P. (1994). Competitiveness: A Dangerous Obsession. Foreign Affairs, 73(2), 28-44.

Krugman, P. R. (1996). Making sense of the competitiveness debate. Oxford review of economic policy, 12(3), 17-25.

Leydesdorff, L. (2001) Scientometrics. En: N. J. Smelser and P. B. Baltes (Editores), International Encyclopedia of the Social and Behavioral Sciences, Oxford; Pergamon, (pp. 13752-13755).

Martinez, E. (2008). La competitividad regional y urbana dentro de la agenda territorial europea. XI Jornadas de Economía Crítica, Bilbao.

Merton, R. K. (1996). On Social Structure and Science. Chicago: University of Chicago Press.

Nisonger, T.E. (2001) Report on the 8th international conference on scientometrics and informetrics in Sydney, Australia. Library Collections, Acquisitions, and Technical Services, 25(4), 485-488.

Pearce, D (1995) Economía de los Recursos Naturales y el Medio Ambiente. Colegio de Economistas de Madrid. Celeste: Madrid.

Porter, M. E. (1985). Competitve advantage. Creating and Sustaining Superior Performance. New York: Free Press.

Presley, R.L; Caraway, B.L (1999) An Interview with Eugene Garfield Serials Review 25(3).

Storper, M. (1997). The regional world: territorial development in a global economy. New York: Guilford Press.

Sydney, Australia Library Collections, Acquisitions, and Technical Services, 25(4), 485-488.

Teece, D. J., Pisano, G. and Shuen, A. (1997), Dynamic capabilities and strategic management. Strat. Mgmt. J., 18: 509-533. 